\title{
The VINCIA Antenna Shower for Hadron Colliders
}

\section{Peter Skands*}

School of Physics and Astronomy, Monash University, VIC-3800, Australia

E-mail: peter.skands@monash.edu

\section{Nadine Fischer}

School of Physics and Astronomy, Monash University, VIC-3800, Australia

\section{Stefan Prestel}

SLAC National Accelerator Laboratory, Menlo Park, CA 94025, USA

\section{Mathias Ritzmann}

Nikhef, Theory Group, Science Park 105, 1098 XG Amsterdam, The Netherlands

\begin{abstract}
We summarise the main features of VINCIA's antenna-based treatment of QCD initial- and finalstate showers, which includes iterated tree-level matrix-element corrections and automated evaluations of perturbative shower uncertainties. The latter are computed on the fly and are cast as a set of alternative weights for each generated event. The resulting algorithm has been made publicly available as a plug-in to the PYTHIA 8 event generator.
\end{abstract}

38th International Conference on High Energy Physics 3-10 August 2016

Chicago, USA

${ }^{*}$ Speaker. 


\section{Introduction}

Monte Carlo event generators [1,2] provide computer-simulated "events", analogous to those produced in real-world high-energy particle collisions. As such, MC generators represent the most detailed - if not always the most accurate - theoretical reference calculations in particle physics, and the play crucial roles in a wide range of contexts, from explicit tests of competing models of dynamical phenomena, to evaluating the effects of various types of corrections or cuts and gauging the sensitivity of proposed physical observables to salient model parameters.

From an author perspective, the fidelity of the physics modelling hinges on two main aspects: the reliability of the approximations made in the various stages of the calculation/simulation, and the extent to which all relevant physical phenomena are accounted for. To some extent, these are of course two sides of the same coin: which effects may be assumed to be negligible, and what level of precision is achieved for those that are not? To complicate matters, the answer to both of these questions tends to depend on which observable is being computed, something which the MC generator itself cannot control; the user may choose to evaluate any (set of) observables on the generated events, from highly inclusive total cross sections, to the most exclusive (even infraredsensitive) hadron-level observables. A "good" event generator is expected to provide a "reasonable approximation" for the full range of possible observables.

General-purpose event generators, like PYTHIA [3], HERWIG [4], and SHERPA [5], employ an ordered, top-down approach, starting with the hardest (short-distance), perturbative physics, and ending with the softest (long-distance) effects of hadronisation and hadron decays. This ordering reflects that the long-distance physics is "seeded" by the short-distance physics, from which it also factorises in the limit of infinite scale separation. Following a hard, perturbative process, MC generators add the effects of:

- Initial- and final-state radiation (ISR \& FSR showers);

- (Sequential) resonance decays (decays of top quarks, Z/W/H bosons, and BSM particles);

- Soft physics: underlying event, hadronisation, hadron and $\tau$ decays, beam remnant fragmentation, colour reconnections, Bose-Einstein correlations, etc.

In this work, we review the recent development of a new formalism for ISR \& FSR showers, implemented in the publicly available VINCIA code [6], which is written as a plug-in to the PYTHIA 8 event generator [3].

\section{VINCIA's Antenna Showers}

Whereas traditional parton showers, such as those implemented in the standalone version of PYTHIA [7, 8], are based on an iterated sequence of $1 \rightarrow 2$ splittings, supplemented to include 4-momentum conservation, running couplings, and (approximately) the effects of QCD coherence, VINCIA is based on the QCD antenna formalism [9, 10, 11, 12, 13], which in the MC shower context was originally pioneered by the ARIADNE program $[10,14]$. In this formalism, splittings are regarded as occurring inside colour-connected pairs of partons called "antennae" (or "Lund dipoles"). The associated $2 \rightarrow 3$ antenna radiation functions reduce to the standard DGLAP kernels 
in the collinear limits, but importantly they also reproduce the soft eikonal factors in the limit of soft-gluon emission, which assures coherence at the leading-colour level independently of the choice of evolution variable and without having to impose any additional vetos on emission angles. The corresponding $2 \rightarrow 3$ antenna phase-space mappings represent exact, on-shell factorisations of the $(n+1) / n$-parton phase spaces. They are Lorentz invariant, conserve 4-momentum, and are valid over all of phase space, not just in the limits. Relative to ARIADNE, the new elements introduced in VINCIA are:

- Iterated matrix-element corrections (MECs), based on and extending the formalism developed in $[15,16,17]$. In the current version of the VINCIA code, these corrections are included up to $\mathscr{O}\left(\alpha_{s}^{3}\right)$ for $p p \rightarrow Z / W / H+$ jets and up to $\mathscr{O}\left(\alpha_{s}^{4}\right)$ for $Z / W / H \rightarrow$ jets and for $p p \rightarrow$ jets, with matrix elements generated by MADGRAPH $[18,19]$.

- Backwards antenna evolution for ISR, based on and extending the formalism developed in $[15,20,21]$. In the current implementation, branchings in initial-initial antennae (i.e., where both of the parent partons are incoming) do impart a transverse recoil to the hard system (essential, e.g., to build up the transverse-momentum spectrum of the lepton pair in $p p \rightarrow$ $\left.Z / \gamma^{*} \rightarrow \ell^{+} \ell^{-}\right)$, while branchings in initial-final antennae do not. We regard the latter as a shortcoming, necessary to obtain tractable expressions for this first version of VINCIA for hadron colliders, and to be improved upon in future work.

- Automated shower uncertainty estimates, formulated as a set of alternative weights for each event, which are calculated on the fly, representing variations of renormalisation scales, nonsingular terms in the antenna functions, colour factors, etc. This is based on the formalism proposed by us in [17], which was recently extended also to standalone PYTHIA [22] (accompanied by an all-orders proof of the method), with similar implementations having appeared in HERWIG [23] and SHERPA [24].

- Smooth ordering; based on the proposal by us [17] to allow branching sequences that are not strongly ordered in the evolution variable, suppressed by an additional power of $1 / Q^{2}$.

The choice of evolution variable, $Q$, is taken to be inversely proportional to the leading singularities of the relevant antenna functions; $p_{\perp}$ for gluon emissions, and virtuality for processes that only contain a single pole (such as $g \rightarrow q \bar{q}$ splittings). In [25], the former was shown to correctly reproduce the logarithms of the virtual $\mathscr{O}\left(\alpha_{s}^{2}\right)$ antenna functions for $q \bar{q} \rightarrow q g \bar{q}$.

The iterated application of matrix-element corrections (MECs) is illustrated schematically in fig. 1. Starting from a Born-level matrix element (squared) for an arbitrary final state, $F$, the antenna shower is used to generate an LL approximation to the matrix element for $F+1$ partons,

$$
\left|M_{F+1}\right|^{2} \stackrel{L L}{\sim} \sum_{i \in \text { ant }} a_{i}\left|M_{F}\right|^{2},
$$

with $a_{i}$ the appropriate antenna functions, including coupling and colour factors. Due to the clean antenna phase-space factorisation, no Jacobian factors appear in this expression, and all the momenta appearing in both $M_{F}$ and $M_{F+1}$ are on shell. The probability to accept the branching is then reweighted by the MEC factor,

$$
P_{\mathrm{MEC}}=\frac{\left|M_{F+1}\right|^{2}}{\sum a_{i}\left|M_{F}\right|^{2}}
$$



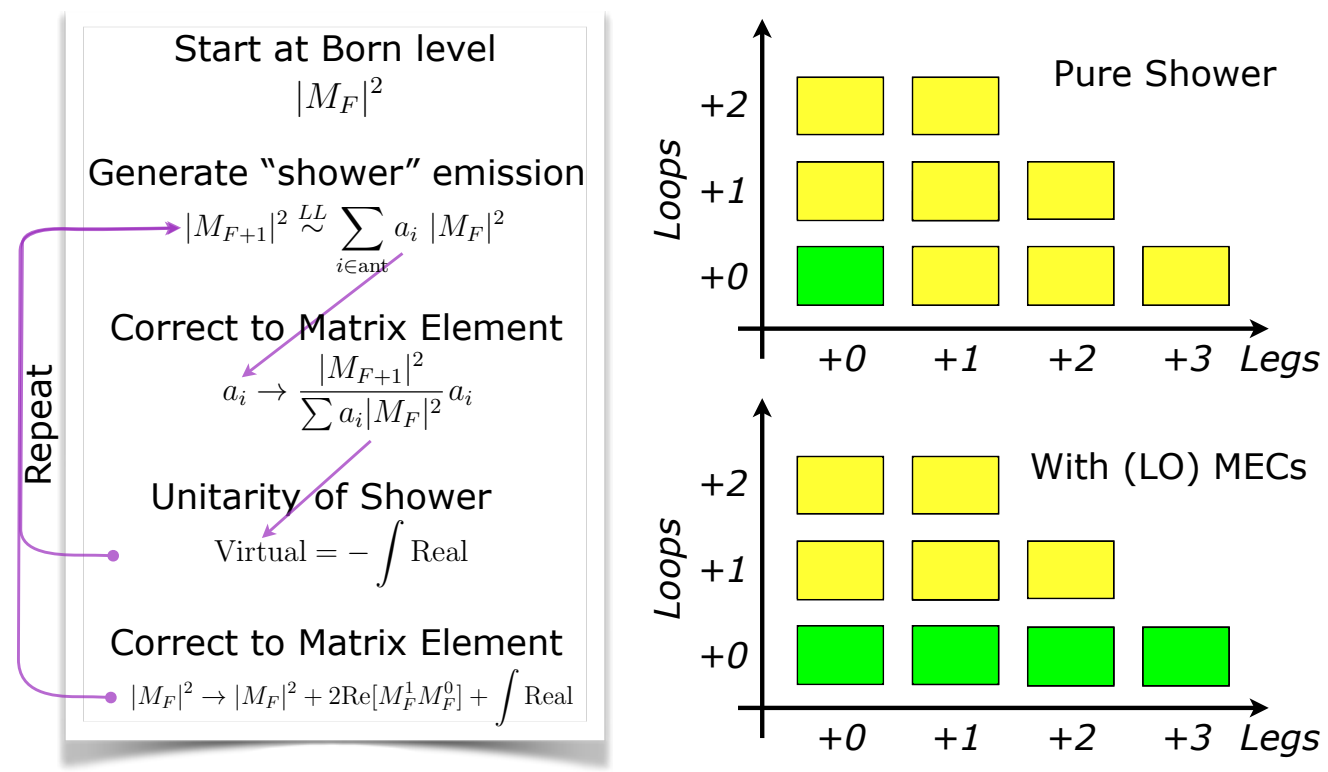

Figure 1: Illustration of the iterative application of matrix-element corrections (MECs) in VINCIA's shower evolution. In the right-hand column, yellow boxes represent coefficients computed at leading-logarithmic (LL) accuracy, while green boxes represent the full perturbative matrix element at the given order.

so that the sum of the corrected branching probabilities, $P_{\mathrm{MEC}} \times a_{i}$ reproduces the full (LO) matrix element, $\left|M_{F+1}\right|^{2}$. Again due to the clean phase-space factorisation, and introducing a Markovian definition of the evolution variable [17, 6], this procedure can be iterated for the second and subsequent branchings, until one "runs out" of (LO) matrix elements. As the left-hand column indicates, analogous corrections can also be defined at the loop level, employing unitarity, though this has so far only been done for the specific case of $Z \rightarrow 3$ jets [25]. For the first emission, this formalism reduces to that of PYTHIA for the tree-level correction $[15,16]$, and to that of POWHEG for the loop-level correction [26, 27]. What is unique to VINCIA's approach is that the corrections can be iterated for multiple emissions, as demonstrated in [17, 25, 6].

For further details on VINCIA's shower, matrix-element corrections, and automated uncertainty variations, see [6] and references therein. The code can be downloaded from

$$
\text { http://vincia.hepforge.org }
$$

where an extensive online HTML User Reference is also available.

Acknowledgements: We thank the convenors of the ICHEP strong-interactions session, in particular E. Nurse, for the invitation to present our work. SP is supported by the US Department of Energy under contract DE-AC02-76SF00515. The work of MR was supported by the European Research Council under Advanced Investigator Grant ERC-AdG-228301 and ERC Advanced Grant no. 320651, "HEP- GAME". PS is the recipient of an Australian Research Council Future Fellowship, FT130100744: "Virtual Colliders: high-accuracy models for high energy physics". 


\section{References}

[1] A. Buckley et al., "General-purpose event generators for LHC physics," Phys. Rept. 504 (2011) 145-233, arXiv:1101.2599 [hep-ph].

[2] P. Skands, "Introduction to QCD," in Proceedings, Theoretical Advanced Study Institute in Elementary Particle Physics: Searching for New Physics at Small and Large Scales (TASI 2012): Boulder, Colorado, June 4-29, 2012, pp. 341-420. 2013. arXiv:1207.2389 [hep-ph] .

[3] T. Sjöstrand, S. Ask, J. R. Christiansen, R. Corke, N. Desai, P. Ilten, S. Mrenna, S. Prestel, C. O. Rasmussen, and P. Z. Skands, “An Introduction to PYTHIA 8.2,” Comput. Phys. Commun. 191 (2015) 159-177, arXiv:1410.3012 [hep-ph].

[4] J. Bellm et al., "Herwig 7.0/Herwig++ 3.0 release note," Eur. Phys. J. C76 no. 4, (2016) 196, arXiv:1512.01178 [hep-ph].

[5] T. Gleisberg, S. Hoeche, F. Krauss, M. Schonherr, S. Schumann, F. Siegert, and J. Winter, "Event generation with SHERPA 1.1," JHEP 02 (2009) 007, arXiv:0811.4622 [hep-ph ].

[6] N. Fischer, S. Prestel, M. Ritzmann, and P. Skands, "Vincia for Hadron Colliders," arXiv:1605.06142 [hep-ph].

[7] T. Sjöstrand and P. Z. Skands, "Transverse-momentum-ordered showers and interleaved multiple interactions,” Eur. Phys. J. C 39 (2005) 129, arXiv: hep-ph/0408302 [hep-ph] .

[8] R. Corke and T. Sjöstrand, “Interleaved Parton Showers and Tuning Prospects,” JHEP 03 (2011) 032, arXiv:1011.1759 [hep-ph].

[9] Y. I. Azimov, Y. L. Dokshitzer, V. A. Khoze, and S. I. Troian, "The String Effect and QCD Coherence," Phys. Lett. B 165 (1985) 147-150.

[10] G. Gustafson and U. Pettersson, “Dipole Formulation of QCD Cascades,” Nucl. Phys. B 306 (1988) 746.

[11] D. A. Kosower, “Antenna factorization of gauge theory amplitudes," Phys. Rev. D 57 (1998) 5410-5416, arXiv:hep-ph/9710213 [hep-ph].

[12] A. Gehrmann-De Ridder, T. Gehrmann, and E. W. N. Glover, “Antenna subtraction at NNLO,” JHEP 09 (2005) 056, arXiv: hep-ph/0505111 [hep-ph] .

[13] W. T. Giele, D. A. Kosower, and P. Z. Skands, “A simple shower and matching algorithm,” Phys. Rev. D 78 (2008) 014026, arXiv:0707.3652 [hep-ph] .

[14] L. Lönnblad, "ARIADNE version 4: A Program for simulation of QCD cascades implementing the color dipole model," Comput. Phys. Commun. 71 (1992) 15-31.

[15] T. Sjöstrand, “A Model for Initial State Parton Showers,” Phys. Lett. B 157 (1985) 321.

[16] E. Norrbin and T. Sjöstrand, “QCD radiation off heavy particles,” Nucl. Phys. B 603 (2001) 297-342, arXiv:hep-ph/0010012 [hep-ph].

[17] W. T. Giele, D. A. Kosower, and P. Z. Skands, "Higher-Order Corrections to Timelike Jets," Phys. Rev. D 84 (2011) 054003, arXiv:1102.2126 [hep-ph].

[18] J. Alwall, P. Demin, S. de Visscher, R. Frederix, M. Herquet, F. Maltoni, T. Plehn, D. L. Rainwater, and T. Stelzer, "MadGraph/MadEvent v4: The New Web Generation," JHEP 09 (2007) 028, arXiv:0706.2334 [hep-ph]. 
[19] J. Alwall, M. Herquet, F. Maltoni, O. Mattelaer, and T. Stelzer, "MadGraph 5 : Going Beyond," JHEP 06 (2011) 128, arXiv:1106.0522 [hep-ph] .

[20] J.-C. Winter and F. Krauss, "Initial-state showering based on colour dipoles connected to incoming parton lines," JHEP 07 (2008) 040, arXiv:0712.3913 [hep-ph ] .

[21] M. Ritzmann, D. A. Kosower, and P. Skands, “Antenna Showers with Hadronic Initial States," Phys. Lett. B 718 (2013) 1345-1350, arXiv:1210.6345 [hep-ph] .

[22] S. Mrenna and P. Skands, "Automated Parton-Shower Variations in Pythia 8," Submitted to: Phys. Rev. D (2016), arXiv:1605.08352 [hep-ph].

[23] J. Bellm, S. Plätzer, P. Richardson, A. Siódmok, and S. Webster, "Reweighting Parton Showers," Phys. Rev. D94 no. 3, (2016) 034028, arXiv:1605.08256 [hep-ph] .

[24] E. Bothmann, M. Schönherr, and S. Schumann, "Reweighting QCD matrix-element and parton-shower calculations," arXiv:1606.08753 [hep-ph] .

[25] L. Hartgring, E. Laenen, and P. Skands, “Antenna Showers with One-Loop Matrix Elements,” JHEP 10 (2013) 127, arXiv:1303.4974 [hep-ph] .

[26] P. Nason, “A New method for combining NLO QCD with shower Monte Carlo algorithms," JHEP 11 (2004) 040, arXiv:hep-ph/0409146 [hep-ph].

[27] S. Frixione, P. Nason, and C. Oleari, "Matching NLO QCD computations with Parton Shower simulations: the POWHEG method," JHEP 11 (2007) 070, arXiv:0709.2092 [hep-ph]. 